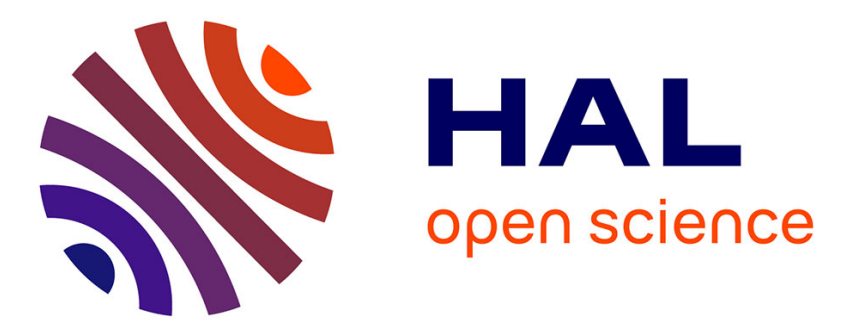

\title{
Une maladie systémique mimant une polyarthrite rhumatoïde
}

Mélanie Legrand, Mathilde Nouvier, L. Paulin, B. Deschamps, Jean-Baptiste Pialat, Fabienne Coury

\section{- To cite this version:}

Mélanie Legrand, Mathilde Nouvier, L. Paulin, B. Deschamps, Jean-Baptiste Pialat, et al.. Une maladie systémique mimant une polyarthrite rhumatoïde. La Revue de Médecine Interne, 2019, 40 (12), pp.831-836. 10.1016/j.revmed.2019.07.015 . hal-02929296

\section{HAL Id: hal-02929296 \\ https://hal.science/hal-02929296}

Submitted on 28 Sep 2021

HAL is a multi-disciplinary open access archive for the deposit and dissemination of scientific research documents, whether they are published or not. The documents may come from teaching and research institutions in France or abroad, or from public or private research centers.
L'archive ouverte pluridisciplinaire HAL, est destinée au dépôt et à la diffusion de documents scientifiques de niveau recherche, publiés ou non, émanant des établissements d'enseignement et de recherche français ou étrangers, des laboratoires publics ou privés. 


\title{
Une maladie systémique mimant une polyarthrite rhumatoïde
}

\section{A systemic disease mimicking a rheumatoid arthritis}

\author{
M. Legrand ${ }^{\mathrm{a}, \mathrm{b}, *}$, M. Nouvier ${ }^{\mathrm{c}}$, L. Paulin ${ }^{\mathrm{a}}$, B. Deschamps ${ }^{\mathrm{a}, \mathrm{b}}$, J.B. Pialat ${ }^{\mathrm{b}, \mathrm{d}, \mathrm{e}}$, F. Coury ${ }^{\mathrm{a}, \mathrm{b}}$ \\ a Service de rhumatologie, hospices civils de Lyon, Centre Hospitalier Lyon Sud, 69495 Pierre-Bénite, France \\ b Université Lyon 1, 69007 Lyon, France \\ ' Service de néphrologie, hospices civils de Lyon, Centre Hospitalier Lyon Sud, 69495 Pierre-Bénite, France \\ d Service d'imagerie médicale et interventionnelle, hospices civils de Lyon, Centre Hospitalier Lyon Sud, 69495 Pierre-Bénite, France \\ e Service d'imagerie médicale et interventionnelle, Hospices civils de Lyon, hôpital Édouard-Herriot, 69003 Lyon, France
}

Introduction. - Des maladies systémiques rares et graves comme l'amylose peuvent mimer un rhumatisme inflammatoire. Il est important de les rechercher, du fait de leur pronostic péjoratif. Nous rapportons le cas d'une amylose AL diagnostiquée à tort comme une polyarthrite rhumatoïde.

Observation. - Une patiente de 71 ans était adressée pour une polyarthrite rhumatoïde séronégative, résistante à trois biothérapies. Elle présentait également une gammapathie monoclonale de signification indéterminée (MGUS) IgA lambda. Secondairement, la patiente développait une protéinurie glomérulaire, en lien avec une amylose AL (Amyloid Light-chain) prouvée sur des biopsies de graisse abdominale et des glandes salivaires accessoires. Avec un traitement par bortezomib-cyclophosphamide-dexamethasone, une rémission hématologique, rénale et articulaire était obtenue, mais une rechute hématologique et articulaire survenait 10 mois après l'interruption de ce traitement.

Conclusion. - L'arthropathie de l'amylose AL est sous-diagnostiquée. Dans une revue d'arthropathie amyloïde associée à un myélome multiple, 33 \% des patients avaient eu un diagnostic erroné de polyarthrite rhumatoïde.

Introduction. - Rare systemic diseases such as amyloidosis can mimic inflammatory rheumatic diseases. Because of their poor prognosis, physicians should rule them out at the onset of inflammatory rheumatism. We report a case of AL amyloidosis misdiagnosed as rheumatoid arthritis.

Case report. - A 71-year-old woman was referred for seronegative rheumatoid arthritis, resistant to three biologic therapies. She had an IgA lambda monoclonal gammopathy of undetermined significance (MGUS). The patient subsequently developed glomerular proteinuria. Abdominal fat and accessory salivary glands biopsies revealed amyloid light-chain (AL) amyloidosis. Treatment with bortezomib-cyclophosphamide-dexamethasone, led to complete hematologic, renal and rheumatologic remission. Ten months after treatment interruption, the patient had an articular and hematologic relapse.

Conclusion. - Amyloid light-chain amyloidosis arthropathy is probably underdiagnosed. A review of amyloid arthropathy associated with multiple myeloma found that $33 \%$ of patients had been misdiagnosed with rheumatoid arthritis.

\footnotetext{
* Auteur correspondant. Service de rhumatologie, Centre Hospitalier Lyon Sud, 165, chemin du Grand Revoyet, 69495 Pierre-Bénite, France. Adresse e-mail : melanie.legrand@chu-lyon.fr (M. Legrand).
} 
La polyarthrite rhumatoïde (PR) est le diagnostic le plus fréquemment porté devant un tableau de polyarthrite bilatérale et symétrique débutant chez un adulte d'âge mûr. En pratique, il est commun de poser ce diagnostic, même en l'absence de facteur rhumatoïde (FR) et/ou d'anticorps anti-peptides citrullinés (ACPA) [1]. Cependant, d'autres maladies systémiques peuvent mimer un rhumatisme inflammatoire [2]. Au diagnostic de rhumatisme inflammatoire, il est primordial d'envisager les diagnostics différentiels tels que les connectivites ou autres maladies systémiques, du fait de leur pronostic potentiellement péjoratif [3]. Dans cette observation, nous rapportons le cas d'une amylose AL diagnostiquée à tort comme une polyarthrite rhumatoïde.

\section{Observation}

Une patiente de 71 ans était hospitalisée en rhumatologie, pour un tableau de polyarthrite qui évoluait depuis quatre ans et résistait au méthotrexate et à plusieurs lignes de biothérapie (adalimumab, abatacept, tocilizumab).

La patiente avait pour antécédents une hypertension artérielle, un cancer de la thyroïde traité par thyroïdectomie et iodothérapie ainsi qu'une gammapathie monoclonale de signification indéterminée (MGUS) IgA $\lambda$.

L'examen clinique à l'admission retrouvait des gonflements articulaires des poignets et des genoux, sans signes extra-articulaires.

La biologie révélait un syndrome inflammatoire marqué (CRéactive Protéine - CRP - à $101 \mathrm{mg} / \mathrm{L}$, vitesse de sédimentation à la première heure à $101 \mathrm{~mm}$, protéine sérique amyloïde $\mathrm{A}$ SAA- à $97 \mathrm{mg} / \mathrm{L}$ pour une norme $<5$, et anémie microcytaire avec une hémoglobine à $9,3 \mathrm{~g} / \mathrm{dL}$ ). La recherche d'auto-anticorps était négative (facteur rhumatoïde, anticorps antipeptides cycliques citrullinés, anticorps anti-nucléaires, anticorps anti-cytoplasme des polynucléaires neutrophiles), de même que la recherche de cryoglobulinémie. Le pic monoclonal IgA $\lambda$ était à 5,6 g/L. Le taux de chaînes légères libres sériques $\lambda$ était augmenté en comparaison à 2012 (308 vs $210 \mathrm{mg} / \mathrm{L}$-norme 5,71-26,3 $\mathrm{mg} / \mathrm{L}$ ) mais avec un ratio $\kappa / \lambda$ normal (Tableau 1 ). La ponction articulaire de genou retrouvait un liquide articulaire inflammatoire ( 5068 globules blancs $/ \mathrm{mm}^{3}$ ) à prédominance de polynucléaires neutrophiles ( $78 \%$ ), stérile et sans cristaux.

Les radiographies des mains, des pieds, des genoux, du rachis et des articulations sacro-iliaques ne retrouvaient pas d'érosion osseuse, il n'y avait pas d'argument pour une chondrocalcinose articulaire ni pour une spondyloarthrite. L'échographie des mains révélait une synovite carpienne bilatérale grade 3 OMERACT. Une Imagerie par Résonance Magnétique (IRM) réalisée au diagnostic objectivait des synovites bilatérales des carpes, des articulations métacarpophalangiennes (MCP) et interphalangiennes proximales (IPP), ainsi que des ténosynovites des fléchisseurs du pouce et des doigts (Fig. 1). La tomographie à émission de positrons (TEP) couplée au scanner montrait un hypermétabolisme intense des ceintures scapulaire et pelvienne, des coudes, des poignets, des genoux et de la hanche droite avec des épanchements intraarticulaires, l'ensemble compatible avec une PR (Fig. 2).

Le diagnostic de MGUS était retenu devant l'absence d'hypercalcémie, l'absence de lésions ostéolytiques aux radiographies standards et au TEP scanner, l'absence d'insuffisance rénale et de protéinurie, un ratio de chaînes légères $\kappa / \lambda$ dans la norme et un myélogramme retrouvant uniquement $6-8 \%$ de plasmocytes dystrophiques.

Un traitement par rituximab et méthotrexate fut instauré, avec une efficacité modérée. Des infiltrations des poignets et des genoux furent également réalisées, sans grande efficacité.

Un an et demi plus tard, la patiente était prise en charge en néphrologie pour une protéinurie $(1,8 \mathrm{~g} / 24 \mathrm{~h})$ glomérulaire (70\% albumine) associée à une protéinurie de Bence-Jones $\lambda$. Le taux de chaînes légères sériques $\lambda$ était augmenté à $300 \mathrm{mg} / \mathrm{L}$, avec un différentiel de chaînes légères libres (dFLC) à 281,5 mg/L

Tableau 1

Résultats des examens complémentaires.

\begin{tabular}{|c|c|c|c|c|}
\hline & 2012 & Août 2017 (diagnostic positif) & Juin 2018 (fin du traitement) & Mai 2019 (rechute) \\
\hline \multicolumn{5}{|l|}{ Clone } \\
\hline sFLC (mg/L) L (3,3-19,4) & L: 210 & L: 301 & $\mathrm{~L}: 8,07(\mathrm{~N})$ & L: 74,4 \\
\hline $\mathrm{K}(5,7-26)$ & $\mathrm{K}: 22,5$ & K: 19,5 & $\mathrm{~K}: 21,2(\mathrm{~N})$ & $\mathrm{K}: 26,1$ \\
\hline dFLC (L-K) & 187,5 & 281,5 & 13,13 (sFLC normales) & 48,3 \\
\hline Ratio k/L 0,26-1,65 (en & 0,11 & 0,06 & 0,35 (sFLC normales) & 0,35 \\
\hline \multicolumn{5}{|c|}{ l'absence d'insuffisance rénale) } \\
\hline \multicolumn{5}{|l|}{ 0,37-3,1 (en présence } \\
\hline \multicolumn{5}{|l|}{ d'insuffisance rénale) } \\
\hline Calcémie corrigée (mmol/L) & 2,44 & 2,18 & 2,31 & 2,3 \\
\hline Créatininémie ( $\mu \mathrm{mol} / \mathrm{L})$ & 58 & 54 & 62 & 58 \\
\hline Hémoglobine $(\mathrm{g} / \mathrm{dL})$ & 10,4 & 10,1 & 11,1 & 10,5 \\
\hline Lésions ostéolytiques & Absence & Absence & Absence & Absence \\
\hline \multicolumn{5}{|l|}{ Amylose } \\
\hline Protéinurie à la BU & Négative & Positive & NR & NR \\
\hline Protéinurie (g/24H) & ND & 1,8 g/24h BJ: positive Alb $71 \%$ & 1,49 BJ: négative & 1,8 BJ: négative \\
\hline $\begin{array}{l}\text { Troponine hypersensible } \\
(\mu \mathrm{g} / \mathrm{L}) \text { Norme }<0,07\end{array}$ & ND & 0,192 & 0,039 & 0,022 \\
\hline NT-pro BNP (ng/L) & ND & 632 & 392 & ND \\
\hline
\end{tabular}

Norme $<100$

Articulaire

Imagerie articulaire

IRM des mains: synovites Échographie des mains: bilatérales des carpes, des MCP Synovite bilatérale des carpes et IPP. Ténosynovites des grade 3 OMERACT fléchisseurs du pouce et des doigts

Arthralgies, Gonflements Arthralgies et gonflements: Coude droit, poignets, MCP, IPP, genoux

Arthralgies et gonflements: Absence Genoux, poignets
Arthralgies: épaules, coudes, poignets et genoux Gonflements: poignet gauche et genou droit

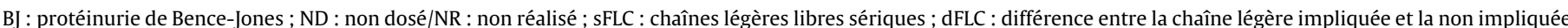

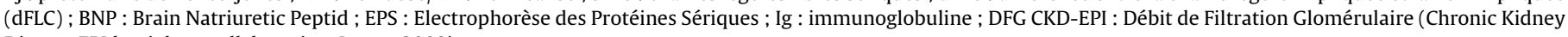
Disease EPIdemiology collaboration, Levey, 2009). 


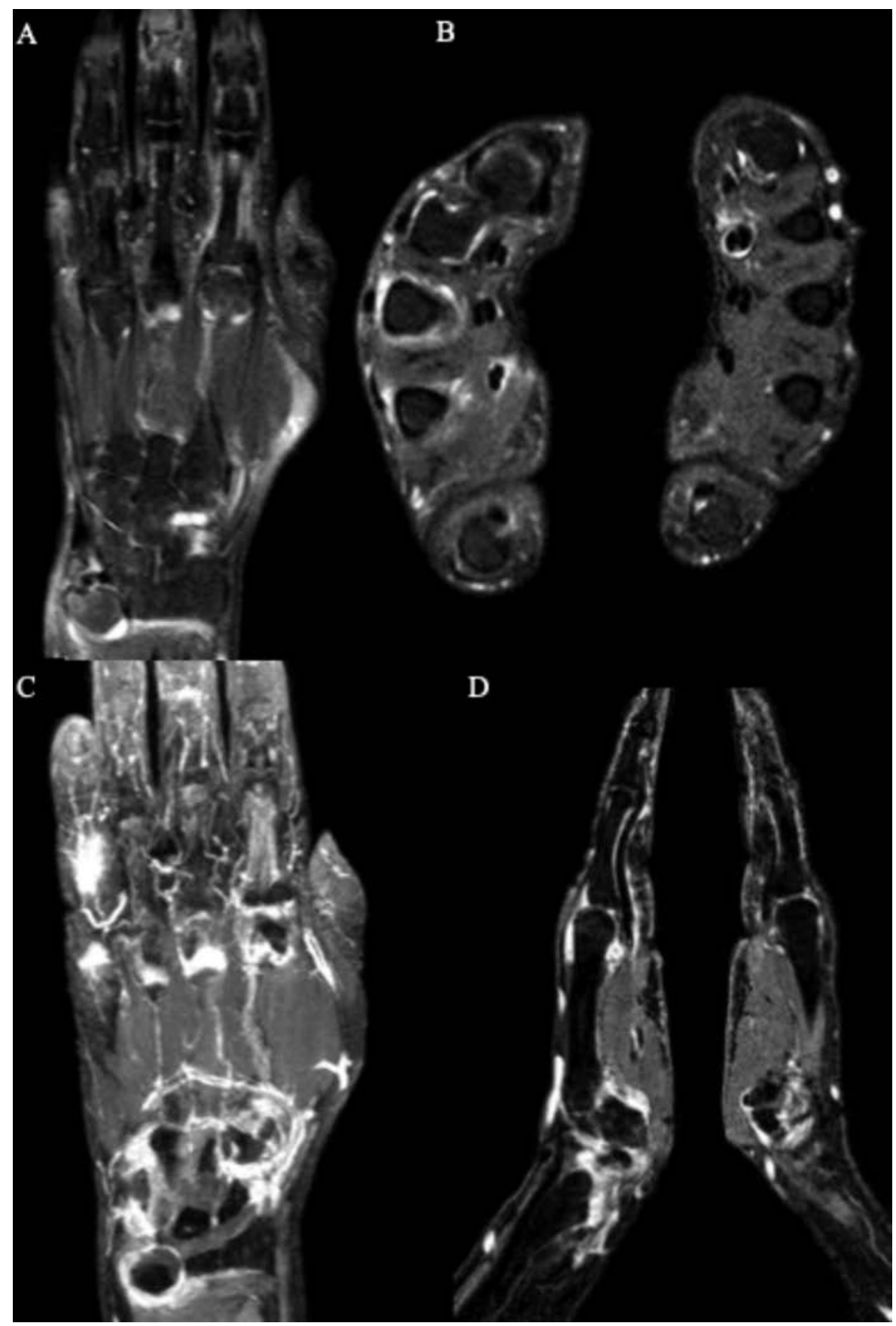

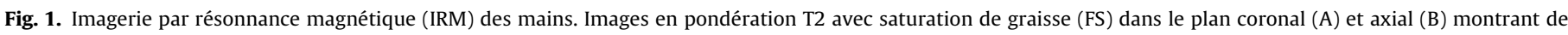

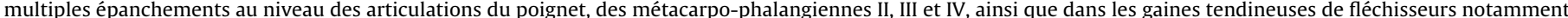

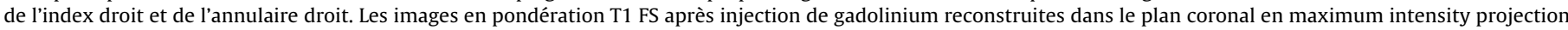

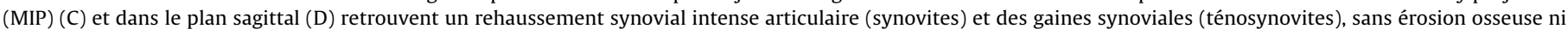
dépôt amyloïde péri-articulaire.

mais un ratio $\kappa / \lambda$ non pathologique à 0,06 . Devant une suspicion de gammapathie monoclonale de signification rénale, une biopsie des glandes salivaires accessoires ainsi qu'une biopsie de la graisse abdominale étaient réalisées [4], mettant en évidence des dépôts amyloïdes en coloration rouge Congo avec en immunofluorescence, une fixation élective de la chaîne $\lambda$ en faveur d'une amylose AL $\lambda$. Les marqueurs cardiaques NT pro BNP et troponine hypersensible étaient élevés, respectivement à $632 \mathrm{pg} / \mathrm{mL}$ et $0,192 \mathrm{ng} / \mathrm{mL}$, correspondant à un score de survie à 2 (NT pro $\mathrm{BNP}<1800 \mathrm{pg} / \mathrm{L}$, troponine $>0,025 \mathrm{ng} / \mathrm{mL}$, différentiel de chaînes légères $>180 \mathrm{mg} / \mathrm{L}$ ). Cependant, il n'existait pas d'atteinte cardiaque sur l'échocardiographie et l'IRM cardiaque ne retrouvait pas non plus d'argument pour une infiltration myocardique amyloïde. La patiente refusait la biopsie synoviale.

Le diagnostic de polyarthrite amyloïde dans le cadre d'une amylose AL accompagnant une gammapathie monoclonale $\operatorname{IgA} \lambda$ était retenu.

Après huit cures de bortezomib-cyclophosphamide-dexamethasone, une réponse hématologique complète était obtenue, définie par la disparition des chaînes légères sériques et urinaires. Les symptômes articulaires disparaissaient rapidement. Dix mois après la fin du traitement, les arthralgies rechutaient, parallèlement à une 


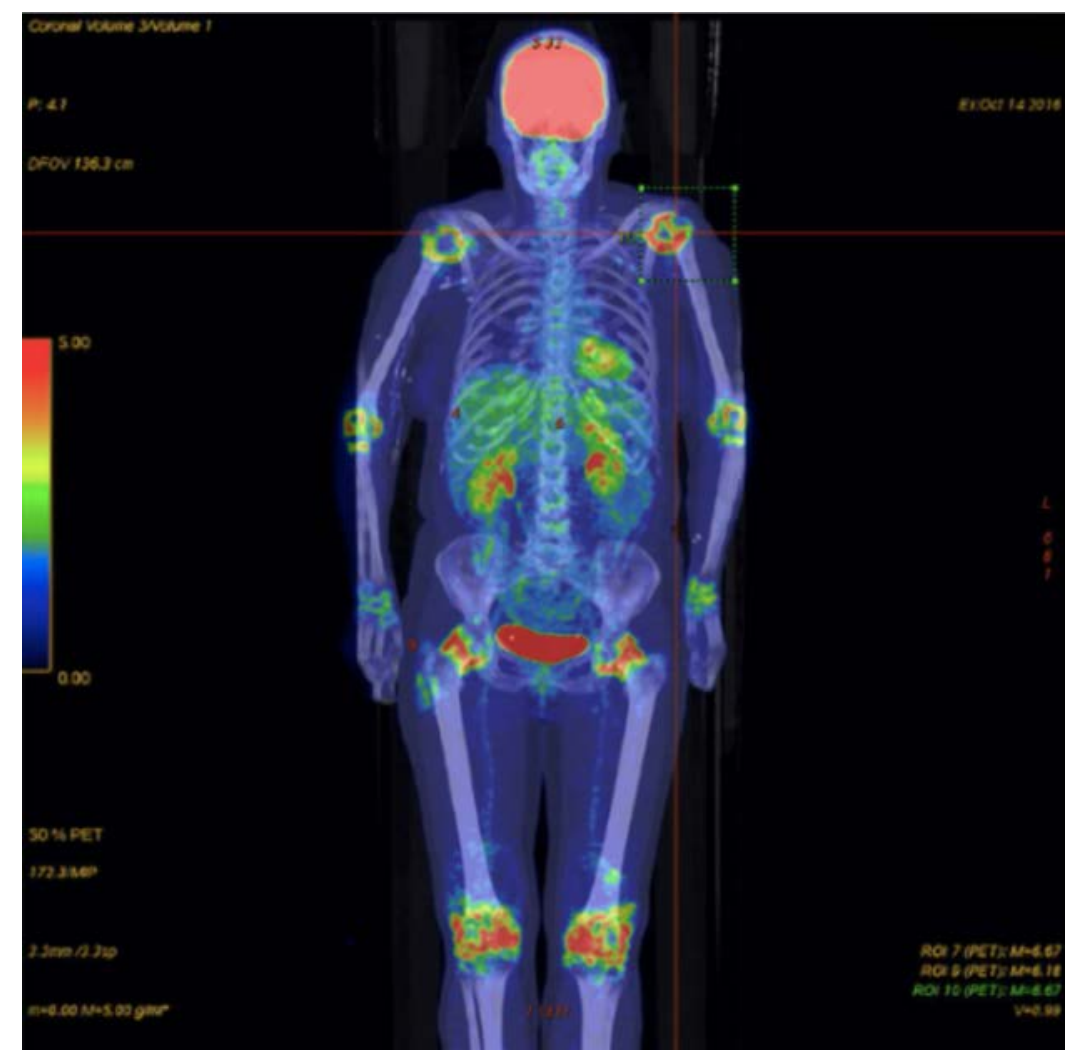

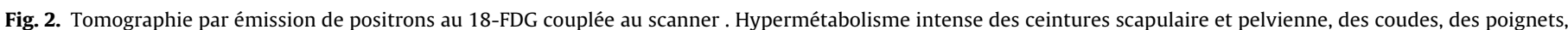
des genoux et des hanches.

réascension du taux des chaînes légères (Tableau 1). Une seconde ligne de traitement par dexamethasone-revlimid était instaurée pour cette rechute articulaire et hématologique.

\section{Discussion}

L'amylose regroupe des maladies rares causées par l'accumulation extracellulaire de protéines à la conformation fibrillaire anormale en feuillets béta-plissés. Les principaux types d'amylose sont définis en fonction des dépôts protéiques amyloïdes observés en histologie : l'amylose AL et l'amylose AA qui sont les plus courantes, l'amylose à béta-2-microglobuline, l'amylose sénile à transthyrétine et enfin les amyloses génétiques [5].

L'amylose AL, à bêta- 2 microglobuline et l'amylose sénile sont responsables de manifestations ostéo-articulaires tandis que l'amylose AA est une amylose de forme extra-articulaire, secondaire à une maladie inflammatoire chronique, un rhumatismes inflammatoire par exemple, et en particulier la PR [6].

L'amylose AL est liée au dépôt extracellulaire de chaînes légères libres (et plus rarement, de chaînes lourdes) d'immunoglobulines monoclonales, produites par une population monoclonale lymphoplasmocytaire [7]. L'amylose AL est l'amylose la plus fréquemment retrouvée dans les pays dits développés, avec une prévalence aux États-Unis estimée à 40,5 cas par million d'habitants en 2015 [8]. En France, son incidence est estimée à 8 à 9 cas par million d'habitants, soit 500 nouveaux cas par an [9]. Elle touche préférentiellement les hommes, avec un âge moyen au diagnostic de 65 ans. L'amylose AL est associée dans $80 \%$ des cas à une MGUS et dans 10 à $20 \%$ des cas à un myélome multiple [2]. Un composant monoclonal sérique ou urinaire est identifié dans 80 à $90 \%$ des cas [10]. Au diagnostic, l'association d'une électrophorèse des protéines sériques et des protéines urinaire, couplée à l'immunofixation, ainsi que le dosage des chaînes légères libres sériques (sFLC), permet d'obtenir l'identification du composant monoclonal circulant avec une sensibilité de $100 \%$ [11].

Le composant monoclonal est le plus souvent une immunoglobuline (Ig) de type $G(24,2 \%)$, suivie du type $A(11,8 \%)$ puis $M(2,9 \%)$. Le plus souvent la chaîne légère est de type $\lambda$ : dans la cohorte japonaise de 741 patients atteints d'amylose AL, une chaîne $\lambda$ était retrouvée chez $78 \%$ des patients [12]. L'amylose AL est une maladie grave, responsable de décès lorsque la prise en charge est tardive, alors que les traitements utilisés (melphalan- dexamethasone, thalidomide, lénalidomide et surtout le bortezomib), efficaces chez la majorité des patients, permettent une rémission hématologique et une médiane de survie prolongée, supérieure à 5 ans. Un diagnostic précoce est donc essentiel [7]. Le pronostic est déterminé par l'atteinte cardiaque, l'importance de la plasmocytose médullaire, le taux sérique de chaînes légères, sFLC, et le dFLC, ainsi que par le nombre d'organes atteints $[11,13,14]$.

La fréquence des atteintes articulaires au cours de l'amylose AL est estimée d'après Gertz et Kyle entre 2 et $5 \%$ [9]. La polyarthrite amyloïde est la manifestation articulaire la plus fréquente (70$80 \%$ des cas). Il s'agit d'une polyarthrite bilatérale et symétrique d'installation progressive $[2,13,15]$. Un gonflement parfois pseudotumoral de la face dorsale des mains et des poignets est parfois noté, résultant d'une infiltration des tissus mous péri-articulaires par les dépôts amyloïdes. Il en résulte une limitation douloureuse de la mobilité des articulations, en particulier au niveau des épaules, poignets, MCP, IPP et genoux. Un signe articulaire quasi pathognomonique de l'amylose AL est l'aspect en épaulette de la ceinture scapulaire, aussi appelé "shoulder pad sign ", souvent associé à une atteinte myocardique [16]. Les autres atteintes articulaires pouvant se rencontrer dans l'amylose AL sont une infiltration des tendons des fléchisseurs des doigts à l'origine d'une attitude irréductible en flexion des doigts, un syndrome du canal carpien (25\%), ainsi que des nodules cutanés en regard des articulations des coudes, des 
poignets ou des doigts $[2,9,15]$. Sur les radiographies standards, les signes d'arthropathie sont souvent absents ou non spécifiques. On peut retrouver une déminéralisation et un épaississement des tissus mous péri-articulaires, en regard des épiphyses. Typiquement, l'interligne articulaire est préservée voire augmentée, du fait de l'accumulation intra-articulaire des dépôts amylö̈des. Rarement, il peut y avoir une atteinte érosive avec des géodes et/ou des érosions sous chondrales finement délimitées par un liseré d'ostéosclérose [2].

Dans cette observation, il n'a malheureusement pas été possible d'apporter la preuve histologique de l'atteinte articulaire de l'amylose $\mathrm{AL}$, du fait du refus de la biopsie par la patiente. Il aurait en effet été intéressant de confirmer la présence de dépôts amyloïdes (par la coloration rouge Congo) et celle de chaînes légères dans ces dépôts (en immunoflurorescence). Cependant, nous avions de solides arguments pour retenir le diagnostic de polyarthrite amyloïde dans le cadre d'une amylose AL. Tout d'abord, on ne retrouvait pas de diagnostic différentiel pour ce rhumatisme articulaire : absence d'autoanticorps, d'hyperuricémie ou de microcristaux dans le liquide synovial du genou, absence d'argument pour une étiologie infectieuse (sérologie VIH, hépatites $B$ et $C$ négatives, liquide articulaire stérile). De même, la sémiologie radiologique était peu en faveur d'une PR : absence de déminéralisation juxta articulaire en bande ou d'érosions osseuses typiques des articulations MCP, des carpes ou de la styloïde ulnaire, conservation des interlignes articulaires. Il n'y avait pas non plus d'argument radiologique pour une pathologie microcristalline en l'absence de calcification ligamentaire ou méniscale ou de tophus osseux. Cliniquement, l'âge de survenue tardif de ce rhumatisme et l'absence de réponse thérapeutique à trois lignes de biothérapie en association au méthotrexate et aux traitements locaux (infiltrations) étaient peu en faveur de ce diagnostic de PR.

Dans la littérature, l'association d'une PR à une amylose AA est relativement fréquente. En revanche, il n'existe aucune donnée, à notre connaissance, concernant l'association d'une PR à une amylose AL. Dans une cohorte japonaise de 872 patients atteints de MGUS, 11 patients avaient une $\mathrm{PR}(1,3 \%)$ [17]. Ce taux est inférieur à la prévalence de la MGUS dans la population générale, estimée à $3 \%$ après 50 ans [18]. Au vu des données de la littérature et du faisceau d'arguments cliniques et biologiques dont nous disposions dans cette observation, le diagnostic de polyarthrite amyloïde était plus probable, qu'une association fortuite entre une PR séronégative et une amylose AL.

Le diagnostic a donc été retenu devant :

- l'absence de diagnostic différentiel ;

- la probabilité élevée de polyarthrite amyloïde en présence d'une amylose AL (confirmée sur les biopsies de graisse abdominale et des glandes salivaires accessoires) ;

- la régression des symptômes articulaires parallèlement à l'obtention de la rémission hématologique avec le traitement par bortezomib- cyclophosphamide- dexamethasone ;

- la rechute des arthralgies parallèlement à la rechute hématologique.

L'instauration précoce d'un traitement de fond avant la survenue de lésions structurales est capitale dans la prise en charge de l'arthrite débutante (PR ou arthrite indifférenciée) avec une fenêtre d'opportunité thérapeutique durant les premiers mois d'évolution de la maladie $[3,19]$. En cas d'arthrite indifférenciée, il est recommandé d'instaurer un traitement de fond le plus rapidement possible, même en l'absence de confirmation du diagnostic, notamment s'il existe des facteurs de risque de progression vers une PR ou une atteinte chronique (niveau d'activité de la maladie élevé, positivité des ACPA et/ou du FR, atteinte structurale) [3,19]. Cet objectif thérapeutique implique un diagnostic précoce de la PR.
L'EUropean League Against Rheumatism (EULAR) a ainsi défini des paramètres devant faire suspecter une PR devant des arthralgies : durée des symptômes inférieure à un an, atteinte des MCP, raideur matinale d'une durée supérieure à 60 minutes, prédominance matinale des symptômes, antécédent familial de PR au premier degré, difficulté à fermer le poing et "squeeze test positif " au niveau des MCP [20]. De plus, l'EULAR et l'American College of Rheumatology (ACR) ont défini des critères de classification pour les arthrites débutantes [21]. Ces critères ne peuvent s'envisager qu'en présence d'au moins une synovite clinique et surtout en l'absence de diagnostic différentiel pouvant expliquer les symptômes. Ces critères ne sont pas des critères diagnostiques mais une aide intéressante pour le diagnostic de PR précoce. Cependant, ils ne doivent pas remplacer le jugement du clinicien, comme le soulignent les recommandations de la Société Française de Rhumatologie (SFR) 2018 sur la PR. Ces recommandations rappellent l'importance de l'appréciation clinique, qui prime sur les critères de classification de l'ACR/EULAR $2010[20,21]$.

De façon curieuse, cette patiente présentait à l'échographie comme à l'IRM (réalisée au diagnostic) des synovites et ténosynovites, et non une hypertrophie des tissus mous ou synoviaux comme habituellement décrite au cours de l'amylose AL. En effet, l'IRM articulaire dans l'amylose AL révèle typiquement des épaississements synoviaux en hyposignal T1 rehaussés après injection de gadolinium et en isosignal T2. Cependant l'atteinte articulaire de l'amylose AL est sous-estimée car sous-diagnostiquée et les données radio-cliniques disponibles sont limitées.

Dans une série de 101 observations de myélome multiple associé à une arthropathie amyloïde de 1931 à 2012, 33 \% des patients avaient eu un diagnostic erroné de PR [22]. Dans cette série, 72 patients (78\% des patients) présentaient une atteinte polyarticulaire de l'amylose. Dans $62 \%$ des cas, l'arthrite était le mode de présentation initial, précédent le diagnostic de myélome d'une médiane de 11 mois. L'atteinte articulaire la plus fréquente était une polyarthrite ( $78 \%$ ) mais $18 \%$ avaient une oligoarthrite et 3,3\% une atteinte monoarticulaire. Les articulations les plus fréquemment touchées étaient les épaules, puis les genoux, les mains et les poignets. Sur le plan de l'imagerie, des lésions ostéolytiques en lien avec l'amylose étaient retrouvées sur des radiographies standards chez seulement cinq patients. Un élargissement de l'interligne articulaire était rapporté dans cinq cas, au niveau gléno-huméral pour quatre d'entre eux et de l'articulation acromio-claviculaire pour un cas. Une inflammation articulaire et péri-articulaire était présente à l'IRM chez six patients, mais des synovites étaient retrouvées uniquement pour deux d'entre eux. Un TEP scanner était disponible chez un seul patient et révélait un hypermétabolisme des articulations symptomatiques. Sur le plan histologique, la revue de 15 biopsies synoviales suggérait une lésion élémentaire commune, caractérisée par une synovite modérée, associée à une infiltration mononucléaire de bas grade.

Il n'existe que très peu de données sur les caractéristiques du liquide articulaire de patients avec polyarthrite amylö̈de. Ici, le liquide articulaire était inflammatoire, avec 5068 globules blancs $/ \mathrm{mm}^{3}$ à prédominance de polynucléaires neutrophiles (78\%). Dans l'article de Gordon et al., sur trois patients ayant une polyarthrite amyloïde, le liquide synovial était inflammatoire avec en moyenne 2460 globules blancs $/ \mathrm{mm}^{3}$ et un infiltrat essentiellement constitué de cellules mononucléées, donc lymphocytaire ou monocytaire [23]. Dans d'autres articles de la littérature [2,24], le liquide synovial observé était non-inflammatoire avec un faible contenu en globules blancs, mais exceptionnellement inflammatoire avec un infiltrat en polynucléaires neutrophiles.

Tout comme la PR, l'arthropathie de l'amylose AL pourrait donc être considérée comme une maladie inflammatoire de la synoviale. La présence de synovites cliniques et l'aspect de l'IRM rapporté 
dans le cas rapporté ici nous paraissent donc compatibles avec cette hypothèse.

Ainsi, il faut rester prudent avant de poser un diagnostic de PR séronégative non érosive et ne pas négliger la recherche de maladies systémiques plus rares, dont le pronostic peut être péjoratif. Les symptômes articulaires peuvent être le mode d'entrée d'une amylose, dont le diagnostic doit être le plus précoce possible. La SFR ne propose pas de façon systématique l'électrophorèse des protéines sériques dans le bilan initial minimal d'une PR, mais souligne la nécessité d'éliminer les diagnostics différentiels par des examens complémentaires. Il est donc primordial de réaliser une électrophorèse des protéines sériques et une recherche de protéinurie à la bandelette urinaire, lors du bilan initial d'un rhumatisme inflammatoire. Nous soulignons également l'importance de la bandelette urinaire au cours du suivi des patients atteints de rhumatismes inflammatoires, afin de dépister une éventuelle protéinurie liée à une amylose, qu'elle soit de type AA ou AL.

\section{Contributions des auteurs}

Recueil des données cliniques, rédaction du case report : M.L.

Néphrologue référent : M.N.

Rhumatologue référent : L. P.

Aide à la rédaction : B.D.

Sélection, traitement des examens des imageries et légendes des figures : J-B.P, M.L.

Supervision de la rédaction, relecture et correction du manuscrit. Suivi du projet : F.C.

\section{Déclaration de liens d'intérêts}

Les auteurs déclarent ne pas avoir de liens d'intérêts.

\section{Références}

[1] Pratt AG, Isaacs JD. Seronegative rheumatoid arthritis: pathogenetic and therapeutic aspects. Best Pract Res Clin Rheumatol 2014;28:651-9.

[2] M'bappé P, Grateau G. Osteo-articular manifestations of amyloidosis. Best Pract Res Clin Rheumatol 2012:26:459-75.

[3] Daien C, Hua C, Gaujoux-Viala C, Cantagrel A, Dubremetz M, Dougados M, et al. Update of French society for rheumatology recommendations for managing rheumatoid arthritis. Joint Bone Spine 2019;86:135-50.

[4] Javaugue V, Bouteau I, Sirac C, Quellard N, Diolez J, Colombo A, et al. Classification et prise en charge thérapeutique des gammapathies monoclonales de signification rénale. Rev Med Interne 2018;39:161-70.
[5] Benson MD, Buxbaum JN, Eisenberg DS, Merlini G, Saraiva MJM, Sekijima $\mathrm{Y}$, et al. Amyloid nomenclature 2018: recommendations by the International Society of Amyloidosis (ISA) nomenclature committee. Amyloid 2018;25: 215-9.

[6] Nakamura T. Amyloid A amyloidosis secondary to rheumatoid arthritis: pathophysiology and treatments. Clin Exp Rheumatol 2011;29:850-7.

[7] Jaccard A, Desport E, Mohty D, Bridoux F. Amylose AL. Rev Med Interne 2015;36:89-97.

[8] Quock TP, Yan T, Chang E, Guthrie S, Broder MS. Epidemiology of AL amyloidosis: a real-world study using US claims data. Blood Adv 2018;2:1046-53.

[9] Gertz MA, Kyle RA. Primary systemic amyloidosis-a diagnostic primer. Mayo Clin Proc 1989;64:1505-19.

[10] Desport E, Bridoux F, Sirac C, Delbes S, Bender S, Fernandez B, et al. AL amyloidosis. Orphanet J Rare Dis 2012;7:54.

[11] Martellosio J-P, Leleu X, Roblot P, Martin M, Puyade M. Dosage des chaînes légères libres : indications et méthodes. Rev Med Interne 2019;40: 297-305.

[12] Shimazaki C, Hata H, Iida S, Ueda M, Katoh N, Sekijima Y, et al. Nationwide Survey of 741 Patients with Systemic Amyloid Light-chain Amyloidosis in Japan. Intern Med 2018;57:181-7.

[13] Gertz MA. Immunoglobulin light chain amyloidosis: 2018 Update on diagnosis, prognosis, and treatment. Am J Hematol 2018;93:1169-80.

[14] Dispenzieri A, Gertz MA, Kyle RA, Lacy MQ Burritt MF, Therneau TM, et al. Prognostication of survival using cardiac troponins and N-termina pro-brain natriuretic peptide in patients with primary systemic amyloidosis undergoing peripheral blood stem cell transplantation. Blood 2004;104: $1881-7$.

[15] Prokaeva T, Spencer B, Kaut M, Ozonoff A, Doros G, Connors LH, et al Soft tissue, joint, and bone manifestations of AL amyloidosis: clinical presentation, molecular features, and survival. Arthritis Rheum 2007;56: 3858-68.

[16] Katz GA, Peter JB, Pearson CM, Adams WS. The shoulder-pad sign-a diagnostic feature of amyloid arthropathy. N Engl J Med 1973;288:354-5.

[17] Yang Y, Chen L, Jia Y, Liu Y, Wen L, Liang Y, et al. Monoclonal gammopathy in rheumatic diseases. Clin Rheumatol 2018;37:1751-62.

[18] Dispenzieri A, Katzmann JA, Kyle RA, Larson DR, Melton LJ, Colby CL, et al. Prevalence and risk of progression of light-chain monoclonal gammopathy of undetermined significance: a retrospective population-based cohort study. Lancet 2010:375:1721-8.

[19] Combe B, Landewe R, Daien CI, Hua C, Aletaha D, Álvaro-Gracia JM, et al. 2016 update of the EULAR recommendations for the management of early arthritis. Ann Rheum Dis 2017;76:948-59.

[20] van Steenbergen HW, Aletaha D, Beaart-van de Voorde LJJ, Brouwer E, Codreanu C, Combe B, et al. EULAR definition of arthralgia suspicious for progression to rheumatoid arthritis. Ann Rheum Dis 2017;76:491-6.

[21] Kay J, Upchurch KS. ACR/EULAR 2010 rheumatoid arthritis classification criteria. Rheumatology (Oxford) 2012;51(Suppl. 6):vi5-9.

[22] Elsaman AM, Radwan AR, Akmatov MK, Della Beffa C, Walker A, Mayer CT, et al. Amyloid arthropathy associated with multiple myeloma: a systematic analysis of 101 reported cases. Semin Arthritis Rheum 2013;43: 405-12.

[23] Gordon DA, Pruzanski W, Ogryzlo MA. Synovial fluid examination for the diagnosis of amyloidosis. Ann Rheum Dis 1973:32:428-30.

[24] Lakhanpal S, Li CY, Gertz MA, Kyle RA, Hunder GG. Synovial fluid analysis for diagnosis of amyloid arthropathy. Arthritis Rheum 1987;30:419-23. 